\title{
Surgeon-performed Ultrasound-guided Needle Biopsy of the Thyroid: A Safe and Effective Diagnostic Procedure
}

\author{
${ }^{1}$ Michael T Law, ${ }^{2}$ Martyn Taylor, ${ }^{3}$ lan C Bennett \\ ${ }^{1}$ Surgical Fellow, Breast and Endocrine Surgery Unit, Princess Alexandra Hospital, Woolloongabba, Brisbane, Australia \\ ${ }^{2}$ Medical Officer, Breast and Endocrine Surgery Unit, Princess Alexandra Hospital, Woolloongabba, Brisbane, Australia \\ ${ }^{3}$ Endocrine Surgeon, Breast and Endocrine Surgery Unit, Princess Alexandra Hospital, Woolloongabba, Brisbane, Australia
}

Correspondence: lan C Bennett, Endocrine Surgeon, Breast and Endocrine Surgery Unit, Silverton Place, 101 Wickham Terrace Brisbane Qld-4000, Brisbane, Australia, e-mail: iancben@bigpond.com

\section{ABSTRACT}

With the ready availability of good quality portable units, office-based ultrasound (US) is increasingly being utilized by endocrine surgeons in their daily practices, particularly in the management of thyroid patients. Ultrasound-guided fine needle aspiration biopsy (US-FNAB) of thyroid lesions is a useful interventional diagnostic technique which can be readily performed by endocrine surgeons as an office-based procedure offering significant convenience for the patient. A retrospective review of US-FNAB's performed in a single surgeon practice between 2006 and 2008 was undertaken particularly assessing the diagnostic outcomes and complication rates. Factors affecting FNAB outcome were tested using Chi-square test and significance defined as $p<0.05$. A total of 128 FNABs were performed on 100 patients in the time period under review. Mean lesion size was $22 \mathrm{~mm}$, and the majority were solid on US. US-FNAB had sensitivity of $85 \%$, accuracy $75 \%$. No complications were reported. A total of $14.8 \%$ of samples were reported as either malignant, atypical or with Hurthle cell findings. In this series of patients, $22 \%$ of cases proceeded to thyroidectomy, $46 \%$ of which yielded malignancy. The nondiagnostic rate was only $5.5 \%$. Solid lesions $<2 \mathrm{~cm}$ were associated with higher nondiagnostic rate $(p=0.04)$. Surgeon-performed thyroid US-FNAB is a safe and effective procedure which compares favorably to radiologist-performed series and offers a convenient 'one-stop' process for patients. In this series, it was associated with a low nondiagnostic rate and a low complication rate. Surgeon-performed US-FNAB techniques can be easily acquired; however, structured training and appropriate credentialing are important for the maintenance of quality assurance standards.

Keywords: Thyroid, Ultrasound, Fine needle aspiration biopsy, Surgeon-performed.

\section{INTRODUCTION}

Fine needle aspiration biopsy (FNAB) is well recognized as an important but safe diagnostic test in the management of thyroid nodules. The development of fine needle aspiration cytology (FNAC) as a diagnostic tool in the workup of tumors, generally, but for thyroid nodules in particular, was greatly enhanced with the development in the 1960s of the specialized Karolinska Institute FNA Cytology Clinic. ${ }^{1}$ Since that era, FNAC has become an integral part of the workup of thyroid nodules, and up until a decade ago was traditionally performed by the palpation technique, which was however associated with a significant nondiagnostic rate reported to be as high as $30 \%{ }^{2}$ In recent years, high frequency B-mode ultrasound (US) evaluation of the thyroid gland has also proven to be a valuable and efficient tool, and its additional employment to guide FNAB of thyroid nodules with greater accuracy and has been associated with a decrease in the nondiagnostic rate and false-negative rate. $^{3}$

Traditionally, ultrasound-guided fine needle aspiration biopsy (US-FNAB) of the thyroid gland has been performed within radiology services by radiologists. However, with more compact and affordable ultrasound machines becoming readily available, US-FNABs are no longer confined to the realm of the radiology department. Endocrine surgeons with appropriate training and experience are increasingly utilizing office ultrasound in the assessment and workup of thyroid patients, and which is recognized as being associated with greater cost efficiencies and greater convenience for patients. ${ }^{4}$

The aim of this study was to perform a retrospective evaluation of the efficacy and safety of surgeon-performed USFNAB of the thyroid in a series of patients attending the private rooms of one endocrine surgeon (IB) who has formal Australian Society of Medicine (ASUM) accreditation ${ }^{5}$ in the use of officebased ultrasound and who has 17 years experience in utilizing ultrasound in the management of endocrine patients.

\section{METHODS}

A retrospective review of the records of all patients who underwent US-FNAB of the thyroid gland in the private practice of a single surgeon (IB) over a 3-year period (2006-2008) was undertaken. Sonographic features of the thyroid lesions, lesion size, cytology results and complications from the procedures were recorded. Any histopathology result was correlated with the cytology result in instances where patients proceeded to thyroidectomy. 
The performance of FNAB was often based on clinical indications, such as for nodules, which demonstrated a dominant size, especially lesions $>1.0$ to $1.5 \mathrm{~cm}$; or nodules which had significantly increased in size as well as those lesions displaying potentially suspicious sonographic features, such as irregular shape, blurred margins, a heterogeneous echo pattern, microcalcifications or hypervascularity. Additional clinical indications included larger painful cystic swellings requiring decompression.

All US-FNABs were performed by a single endocrine surgeon (IB) at a private clinic following routine clinical and ultrasound examinations and as part of the patients' routine workup of abnormal thyroid lesions. Ultrasounds were performed utilizing the Terason $\mathrm{T} 3000$ portable unit with a high frequency 12.5 L linear probe (Teratech Corp, Burlington, MA, USA). US images were obtained and characteristics of lesions documented.

US-FNAB of the area of interest was performed using a standard aspiration technique. A $6 \mathrm{~cm}$ long 22 gauge needle mounted on a $10 \mathrm{ml}$ syringe was generally used in conjunction with a pistol grip holder into which the syringe was fitted (Fig. 1). Under ultrasound control, the needle was advanced into the lesion along the longitudinal axis of the probe with continuous visualization of the needle tip during insertion and sampling on the ultrasound monitor (Figs 2 and 3). With suction applied several passes were made through each of the lesions to maximize the amount of material in the specimen, which was then deposited on two glass slides and spread by the usual smear technique. One of the slides was left to air dry and the other fixed with Cytospray (2.5\% Carbowax in Ethanol, Fronine Pty Ltd, Riverstone, NSW, Australia). Both slides were then inserted into protective containers and sent for cytological examination with a single private pathology service. Cytologists did not attend the biopsies.

The cytology specimens were forwarded to and analyzed by only one private pathology provider and were classified according to the following cytology criteria (Sullivan Nicolaides

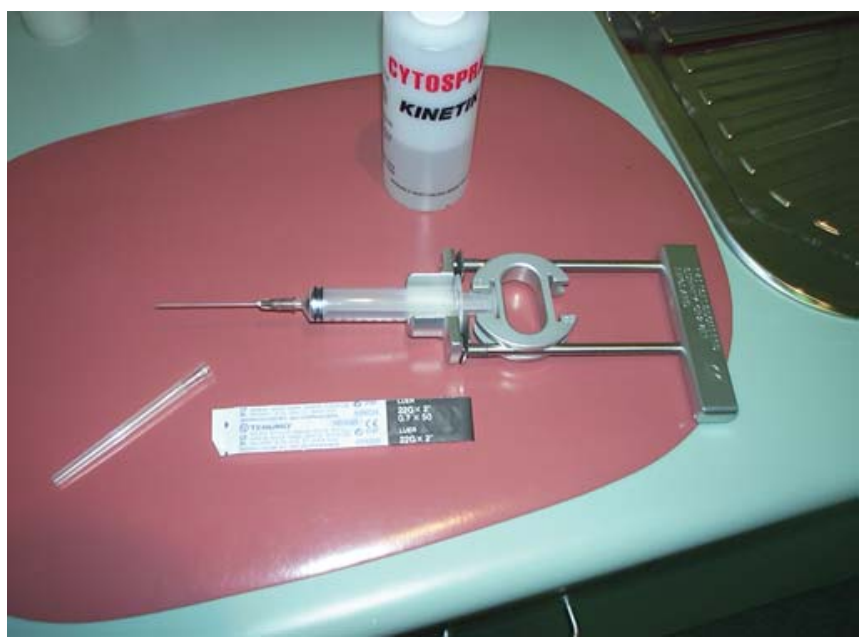

Fig. 1: Equipment useful in the performance of US-FNAB includes the pistol grip syringe holder and $6 \mathrm{~cm} 22$ gauge needle
Pathology, Taringa, QLD, Australia): (1) Nondiagnostic; (2) cyst/cystic degeneration; (3) thyroiditis; (4) benign follicular pattern BFP; (5) atypical follicular pattern AFP; (6) hurthle cell neoplasm and (7) malignant.

Specimens were classified into 'Benign follicular pattern' if they exhibited cytological changes consistent with colloid nodule, nodular hyperplasia and nodular goitre. These corresponded to changes now classified as 'Benign' under the Bethesda classification. ${ }^{6}$ Specimens were deemed inadequate or 'nondiagnostic' if scanty cellularity, no or minimal colloid, poor fixation or poor quality smears were noted.

Sensitivity, specificity, false-negative rates and accuracies were reported for FNAB lesions where surgery was subsequently performed. Factors affecting the FNABs outcomes were tested using Chi-square tests and were considered, if significant and $\mathrm{p}<0.05$.

\section{RESULTS}

Between 2006 and 2008, 128 US-FNABs were performed on 100 patients, thus recording an average of 1.3 FNABs per patient. The proportion of female to male patients was 86 and $14 \%$ respectively, and the overall mean age was 52.8 years (Table 1).

Majority of the lesions were located in the lower poles (81\%) with 13\% occurring in the upper poles and only 6\% were sited in the isthmus. The average lesion size was $22 \mathrm{~mm}$ (range 4-70 mm). On ultrasound, 66\% of lesions biopsied were solid in nature with $34 \%$ being predominately cystic.

Figure 4 demonstrates the breakdown for the results of the cytology analyses for the complete series of FNAB's performed in this review. Overall 102 cases (79.8\%) reported benign cytology (including BFP 47\%, cystic $28.1 \%$ and thyroiditis 4.7\%) and with 19 (14.8\%) biopsies showing atypical, Hurtle cell neoplasm or malignant results. Only seven cases (5.5\%) were reported as nondiagnostic.

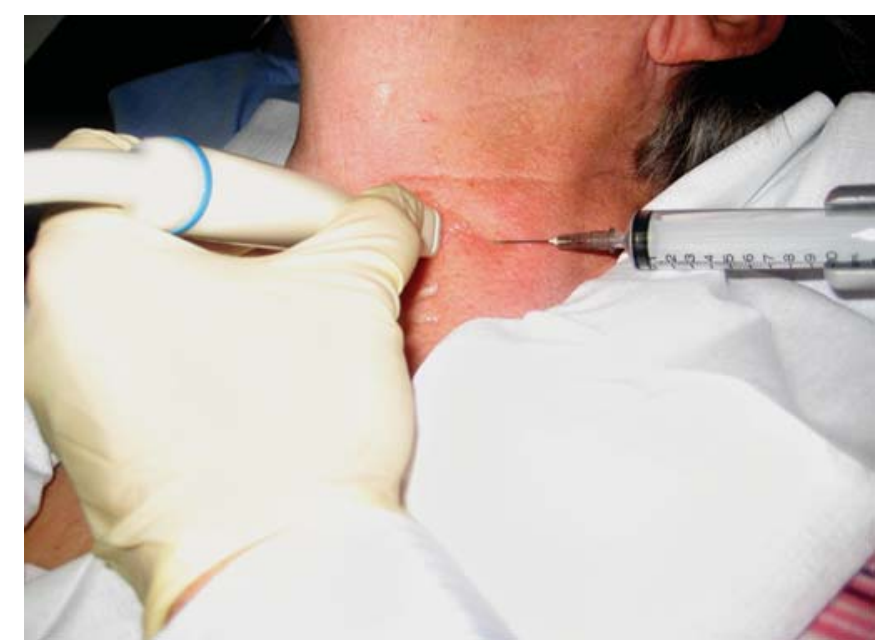

Fig. 2: Holding the US probe over the thyroid, the fine needle is directed into the lesion along the long axis of the probe and a sample is taken under ultrasound visualization 


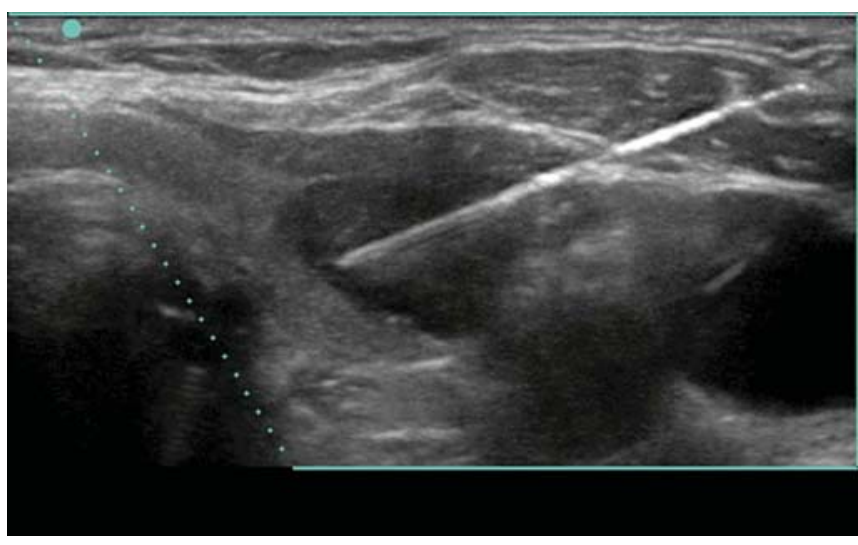

Fig. 3: Ultrasound image of needle tip entering nodule in left lobe thyroid, transverse cross-section

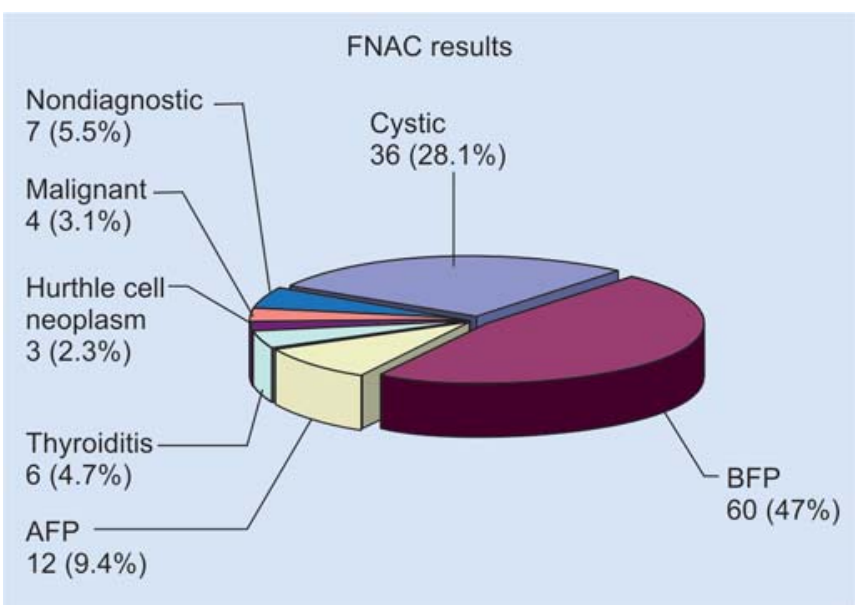

Fig. 4: Cytology results of 128 FNA thyroid biopsies. AFP-atypical follicular pattern; BFP_benign follicular pattern

In terms of the ultrasound appearances, while the usual features of malignancy were looked for such as irregular margins, internal heterogeneity and increased vascularity, there were no particular features which proved more discriminatory than FNAC. In particular, for those 60 patients who returned a BFP result, ultrasound demonstrated completely benign features with 36 (60\%) showing a multinodular pattern; 16

Table 1: Patient and ultrasound features

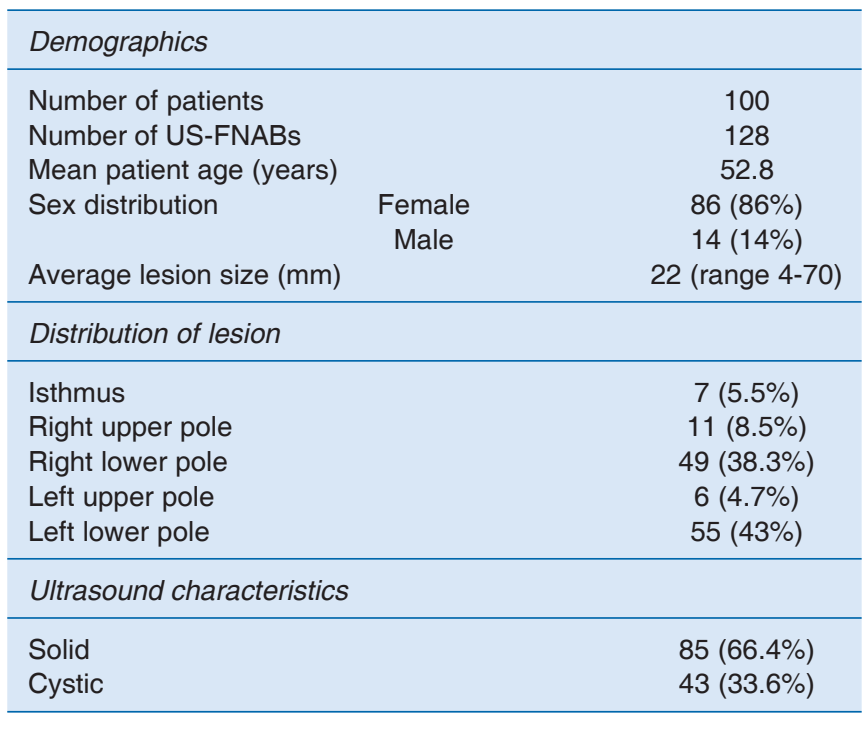

(27\%) being solitary nodules; and 8 (13\%) having an otherwise cystic appearance, and none of the six patients with a BFP result who subsequently underwent surgery were found to have malignancy.

The nondiagnostic rate of US-FNABs correlated primarily with the size of the lesion (Table 2). Lesions less than $21 \mathrm{~mm}$ in size were more likely to yield nondiagnostic results (9.2 vs $5.5 \%, p=0.04$ ). The location of the lesion which may affect the level of difficulties in obtaining adequate material did not appear to affect nondiagnostic rate. In our series, all initially nondiagnostic biopsies were repeated, all of which yielded satisfactory results.

The FNABs were well tolerated and no complications were reported with a follow-up period of 2 to 24 months. There are no known instances of any patient who had benign biopsy result subsequently going on to develop malignancy of that same lesion.

Twenty-eight biopsied lesions proceeded to thyroidectomy (22\%), half of which were total thyroidectomies. All cases showing malignant or atypical cytology results were operated on, and this was the most frequent indication for surgery (57\% of cases). Other indications for surgery included retrosternal goitre, rapid enlargement of a lesion and discordance between cytological and imaging findings.

Of the 28 patients who underwent surgery, malignancy was identified on histology in 13 cases (46.6\%), 12 of which were of papillary carcinomas and one was follicular in type. Sensitivity, specificity, false-negative rate and accuracy of the FNAB in predicting malignancy were 85, 67, 15 and 75\% respectively.

\section{DISCUSSION}

Endocrine surgeons are confronted daily with the problem of managing thyroid nodules which are in fact extremely common, with palpable nodules being present in 4 to $7 \%$ of the population and with over $50 \%$ of adults reported to have thyroid nodules detected on sonography. ${ }^{7}$ The dilemna for the surgeon is the correct management of those nodules which he or she cannot palpate and for which important clinical judgements need to be made. In recent years, both ultrasound and FNAB have proven integral to the safe and efficient management of thyroid nodules providing useful diagnostic imaging criteria and cytological lesion evaluation. Furthermore, ultrasound-guided FNAB has

Table 2: Factors affecting nondiagnostic rate

\begin{tabular}{llcc}
\hline Factors & & $\begin{array}{c}\text { Nondiagnostic } \\
\text { rate }\end{array}$ & p-value \\
\hline \multirow{2}{*}{ Size $(\mathrm{mm})$} & $1-20$ & $9.2 \%$ & 0.04 \\
& $\geq 21$ & $0 \%$ & \\
Location & Isthmus & $0 \%$ & 0.47 \\
& Right upper pole & $8.2 \%$ & \\
& Right lower pole & $0 \%$ & \\
& Left upper pole & $3.6 \%$ & \\
& Left lower pole & $16.7 \%$ & \\
\hline
\end{tabular}


been shown to be more reliable than palpation guided FNAB, with recent literature demonstrating improved sensitivity, specifity and accuracy for US-FNAB compared with palpationguided FNAB. ${ }^{2}$ US-FNAB has also been shown to decrease the rate of nondiagnostic aspirates in patients with small $(<1$ $\mathrm{cm})^{8}$ and cystic nodules. ${ }^{9}$ Generally, speaking FNABs performed by palpation alone carry a nondiagnostic rate of 9 to $32 \%{ }^{2}$ In our series where ultrasound guidance was used, there was a significantly lower nondiagnostic rate of 5.5\%. In a review of US-guided versus palpation FNABs by Morris et $\mathrm{al}^{3}$ USFNAB consistently showed lower nondiagnostic rates in the order of 4 to $21 \%$. This may also translate to a lower surgical intervention rate. Our series reported a surgery rate of $22 \%$, with $46.6 \%$ of those cases yielding malignant histology. This compares favorably to most series of palpation performed FNABs where the malignant yield is often only approximately $20 \% .^{3}$

Traditionally, most US-guided biopsies have been performed by radiologists due to specific sonographic knowledge and access to ultrasound equipment. With the increasing popularity of high quality smaller portable units, more and more US-FNABs are performed by surgeons in the office setting. ${ }^{10}$ Table 3 documents the nondiagnostic rates from various surgeon performed series which range from 3.6 to $13 \%$ which compare favorably to the results of this study and also quite favorably to a series of radiologist performed results with a range of 8 to $23 \% .^{9,11-19}$ It has been recommended that in order to maintain satisfactory standards, US-FNABs should be carried out by dedicated groups of clinicians. ${ }^{20,21}$ Our series demonstrated that with appropriate training, thyroid surgeons are well placed to perform such procedures in the office setting due to their sound understanding of the anatomy and pathology of thyroid gland, and their considerable experience gained from large caseload numbers of patients with thyroid nodules. Although the number of patients who went to surgery in this series was only small, this present study of surgeon-performed US-FNAB has demonstrated a high sensitivity of $85 \%$ and an accuracy of $75 \%$.

Another advantage of surgeon-performed US-FNAB is that of convenience to the patient. Milas ${ }^{22}$ found that patients are generally more satisfied if clinical workup can be performed in a single visit, the so-called 'one-stop shop' scenario.
Furthermore, preoperative surgeon-performed US has the potential to more likely detect other possible pathologies such as parathyroid disease or cervical nodal metastases, thus facilitating appropriate surgical planning. The convenience to patients of being able to have their definitive diagnostic procedure performed at the same occasion as their specialist consultation without the need to visit another clinician or radiology service should not be underestimated. Additionally, the benefits in terms of cost-effectiveness are appreciable. ${ }^{4}$

In this report, the nondiagnostic rate of US-FNABs was shown to be related to the size of the lesion being needled. Smaller lesions have been reported in several series including this one to be associated with higher nondiagnostic rates. ${ }^{23}$ When lesion size was divided into two groups; less than $20 \mathrm{~mm}$ and over $20 \mathrm{~mm}$; a 9.2\% nondiagnostic rate was reported in the former group versus $0 \%$ for the larger lesion $(p=0.04)$ (Table 2). The location of the lesion did not appear to affect nondiagnostic rate although lesions which are more deeply located or in close proximity to vascular structures can pose special technical challenge during biopsy. It has been suggested that the nondiagnostic rate can be reduced by measures such as increasing the number of passes during aspiration ${ }^{24}$ and the selective use of an on-site assessment of cytologists. ${ }^{25}$ However, routine attendance of cytologists is not always necessary. Nondiagnostic rates reported from studies with on-site assessment range from 0.7 to $13 \%,{ }^{25-27}$ whereas the results from other studies without on-site assessment are certainly comparable, and in our series in which on-site cytology was not used the nondiagnostic rate was 5.5\%. On-site cytology availability also requires prior arrangement for cytologists to attend biopsies and hence patients need to set-up additional appointments for dedicated biopsy sessions after their initial assessment, negating the potential convenience afforded by office-based ultrasound. ${ }^{4}$ O'Malley ${ }^{27}$ estimated that biopsies attended by a cytologist took over 3.5 times longer than nonattended ones. However, there may be a role for on-site cytology arrangements for those surgeons who are less experienced and who wish to improve their technique by gaining immediate feedback as to the adequacy of their US-FNAB.

Although the complication rate in relation to US-FNAB is not widely reported, we believe that it is an extremely safe procedure. No complication was recorded in the 128 biopsies

Table 3: Comparison of nondiagnostic rates for radiologist and surgeon-performed US-FNABs

\begin{tabular}{|c|c|c|c|}
\hline Author, year published & Number FNAB's & Nondiagnostic rate (\%) & Operator \\
\hline Khurana, $1998^{11}$ & 119 & 8 & Radiologist \\
\hline Tambouret, $1999^{12}$ & 290 & 15 & Radiologist \\
\hline Mittendorf, $2002^{13}$ & 66 & 23 & Radiologist \\
\hline Mehrotra, $2006^{14}$ & 102 & 17 & Radiologist \\
\hline Sabel, $1998^{9}$ & 79 & 4 & Surgeon \\
\hline Eedes, $2002^{15}$ & 331 & 13 & Surgeon \\
\hline Seiberling, $2008^{16}$ & 271 & 9.4 & Surgeon \\
\hline Bhatki, $2008^{17}$ & 446 & 3.6 & Surgeon \\
\hline Takashima, $1994^{18}$ & 268 & 3.7 & Surg*/Rad\# \\
\hline Carmeci, $1996^{19}$ & 497 & 13.9 & Surg/Rad \\
\hline
\end{tabular}

*Surg: Surgeons performed series; \#Rad: Radiologists performed series 
performed in our series $(0 \%)$. Overall reported complications range from 0 to $8 \% .{ }^{28}$ The most commonly reported complication is hematoma, but other rare complications including needle tract seeding of malignancy, tracheal injury, temporary vocal cord palsy and nodule infarction have also been reported..$^{29}$ Ultrasound guidance has the advantage of being able to identify relations of thyroid lesions to important anatomical structures, such as vessels and trachea, minimizing the chance of inadvertent injuries.

Most thyroid surgeons are familiar with FNABs by palpation technique and proficiency in US-FNAB can be easily acquired through training courses, ${ }^{10,20}$ such as those conducted by Radiological Society of North America and American Association of Clinical Endocrinologists. In Australia, the Australian Society for Ultrasound in Medicine (ASUM) in conjunction with the Royal Australasian College of Surgeons has now established a curriculum and training guidelines for a certificate for clinician performed ultrasound (CCPU) for both breast and endocrine surgeons. ${ }^{5}$

Training workshops in ultrasound are now conducted in Australia subject to the accreditation approval of ASUM. Attendance in structure workshops has been shown to improve operator confidence and skills and minimize complications. ${ }^{30,31}$ Furthermore, structured workshops should form part of the curriculum for training accreditation and recredentialing which is vital in maintaining performance standards in thyroid USFNAB.

\section{CONCLUSION}

Surgeon-performed office-based US-FNAB is an effective diagnostic tool. It has comparable diagnostic success to that of radiologist-performed US-FNAB and is associated with low complication and nondiagnostic rates. It imparts great convenience to both patients and surgeons and is cost-effective. It is easily taught to specialist thyroid surgeons and trainees but structured training and on-going recredentialing are important in maintaining proficiency.

\section{REFERENCES}

1. Lundgren CI, Zedenius J, Skoog L. Fine needle aspiration biopsy of benign thyroid nodules: An evidence-based review. World J Surg 2008;32:1247-52.

2. Cesur M, Corapcioglu D, Bulut S, et al. Comparison of palpationguided fine needle aspiration biopsy to US-guided fine needle aspiration biopsy of the evaluation of thyroid nodules. Thyroid 2006;16:555-61.

3. Morris LF, Ragavendra N, Yeh MW. Evidence-based assessment of the role of ultrasonography in the management of benign thyroid nodules. World J Surg 2008;32:1253-63.

4. Mazzaglia PJ. Surgeon-performed ultrasound in patients referred for thyroid disease improves patient care by minimizing performance of unnecessary procedures and optimizing surgical treatment. World J Surg June 2010;34(6): 1164-70.

5. http://www.asum.com.au/site/index.php Qualifications; CCPU Certificate in Clinician Performed Ultrasound 2010.
6. Baloch ZW, Cibas ES, Clark DP, Layfield LJ, Ljung BM, Pitman MB, et al. The national cancer institute thyroid fine needle aspiration state of the science conference: A summation. Cyto Journal 2008;5(6):1-17.

7. Brander A, Viikinkoski P, Nickels J, Kivisaari L. Thyroid gland: US screening in a random adult population. Radiology 1991;181: 683-87.

8. Reading CC, Charboneau PL, Suen KCH, Thorson SC. Sonographically guided percutaneous biopsy of small masses. AJR 1988;151:189-92.

9. Sabel, MS, Danish H, Velasco J, Staren ED. Use of ultrasoundguided fine needle aspiration biopsy in the management of thyroid disease. The American Surgeon 1998;64:738-42.

10. Charous, SJ. An overview of office-based ultrasonography: New versions of an old technology. Otolaryngology—Head and Neck Surgery 2004;131:1001-03.

11. Khurana KK, Richards VI Chopra PS, et al. The role of ultrasonography-guided fine needle aspiration guided biopsy in the management of nonpalpable and palpable thyroid nodules. Thyroid 1998;8:511-15.

12. Tambouret R, Szyfelbein WM, Pitman MB. Ultrasound-guided fine needle aspiration biopsy of the thyroid. Cancer 1999;87(5):299-305.

13. Mittendorf EA, Tamarkin SW, McHenry CR. The results of ultrasound-guided fine needle aspiration biopsy for evaluation of nodular thyroid disease. Surgery 2002;132(4):648-53; discussion 653-54.

14. Mehrotra P, Viswanathan H, Johnson SJ, et al. Ultrasound guidance improves the accuracy of our preoperative thyroid cytology but not its accuracy. Cytopathology 2006;17: 137-44.

15. Eedes CR, Wang HH. Cost-effectiveness of immediate specimen adequacy assessment of thyroid fine needle aspirations. Am J Clin Pathol 2004;121:64-69.

16. Seiberling KA, Dutra JC, Gunn J. Ultrasound-guided fine needle aspiration biopsy of thyroid nodules performed in the office. The Laryngoscope 2008;118:228-31.

17. Bhatki AM, Brewer B, Robinson-Smith T, Nikiforov Y, Steward DL. Adequacy of surgeon-performed ultrasound-guided thyroid fine needle aspiration biopsy. Otolaryngology-Head and Neck Surgery 2008;139:27-31.

18. Takashima S, Fukuda H, Kobayashi T. Thyroid nodules: Clinical effect of ultrasound-guided fine-needle aspiration biopsy. J Clin Ultrasound 1994;22:535-42.

19. Carmeci C, Jeffrey RB, McDougall IR, Nowels KW, Weigel RJ. Ultrasound-guided fine needle aspiration biopsy of thyroid masses. Thyroid 1998;8:283-89.

20. Ljung BM, Langer J, Mazzaferri EL, Oertel, YC, Wells SA, Waiseman J. Training, credentialing and re-credentialing for the performance of a thyroid FNA. Diagnostic Cytopathology 2008;36:400-06.

21. Suen KC. Fine-needle aspiration biopsy of the thyroid. CMAJ 2002;167:491-95.

22. Milas M, Stephen A, Berber E, Wagner K, Miskulin J, Siperstein A. Ultrasonography for the endocrine surgeon: A valuable clinical tool that enhances diagnostic and therapeutic outcomes. J Surg 2005;138:1193-201.

23. Hatada T, Okada K, Ishii H, Ichii S, Utsunomiya J. Evaluation of ultrasound-guided fine needle aspiration biopsy for thyroid nodules. Am J Surg 1998;175:133-36.

24. Rausch P, Nowels K, Jeffrey RB. Ultrasonographically guided thyroid biopsy: A review with emphasis on technique. J Ultrasound Med 20:79-85. 
25. Baloch ZW, Tam D, Langer J, et al. Ultrasound-guided fine needle aspiration biopsy of the thyroid: Role of on-site assessment and multiple cytologic preparations. Diagn Cytopathol 2000;23:425-29.

26. Nasuti JF, Gupta PK, Baloch ZW. Diagnostic value and costeffectiveness of on-site evaluation of fine needle aspiration specimens: Review of 5,688 cases. Diagn Cytopathol July 2002; 27(1):1-4.

27. O’Malley ME, Weir MM, Hahn PF, et al. US-guided fine needle aspiration biopsy of thyroid nodules: Adequacy of cytologic material and procedure time with and without immediate cytologic analysis. Radiology 2002;222:383-87.
28. Newkirk KA, Ringel MD, Jelinek J, Mark A, Wartofsky L, et al. Ultrasound-guided fine needle aspiration and thyroid disease. Otolaryngol Head Neck Surg 2000;123:700-05.

29. Leung AM, Farwell AP. Unsatisfactory consequences from fine needle aspiration biopsy of thyroid nodules. Thyroid 2008;18(5): 491-92.

30. Law MT, Bennett IC. Structured ultrasonography workshop for breast surgeons: Is it an effective training tool? World Journal of Surgery 2010;34(3):549-54.

31. Hoover SJ, Berry MP, Rossick L, Rege, RV, Jones DB, Ultrasound-guided breast biopsy curriculum for surgical residents. Surgical Innovation 2008;15(1):52-58. 